\title{
XXI. Notes on magnetism.-I. On the energy of magnetized iron
}

\section{Lord Rayleigh Sec. R.S.}

To cite this article: Lord Rayleigh Sec. R.S. (1886) XXI. Notes on magnetism.-I. On the energy of magnetized iron, Philosophical Magazine Series 5, 22:135, 175-183, DOI:

10.1080/14786448608627915

To link to this article: http://dx.doi.org/10.1080/14786448608627915

册 Published online: 08 Jun 2010.

Submit your article to this journal $\lceil\pi$

Џ Article views: 7

Q View related articles $\square$

Citing articles: 1 View citing articles ๘ 


\section{Notes on Magnetism.-I. On the Energy of Magnetized} Iron. By Lord RaYLEIGH, Sec. R.S.*

THE splendid achievements of the last ten years in the practical application of Magnetism have given a renewed impetus to the study of this subject which is sure to bear valuable fruit. Especially to be noted are two memoirs recently published in the 'Philosphical Transactions' of the Royal Society, by Prof. Ewingt, and by Dr. Hopkinsonf, in which are detailed very important data derived from laborious experiment, accompanied by much interesting and suggestive comment.

The results of observation are usually expressed, after the example of Rowland and Stoletow, in the form of curves showing the relation between $\mathfrak{B}$ and $\mathfrak{S}$, the magnetic induction and the magnetizing force. It may be well here to recall the convention in accordance with which $\mathfrak{F}$ is measured. At any point in air, the magnetic force is defined in an elementary manner, and without ambiguity, but when we wish to speak of magnetic force in iron, further explanation is needed. The continuity of the iron is supposed to be interrupted by an infinitely thin crevasse in the interior of which we imagine the measurement to be effected. If the crevasse is parallel to the direction of magnetization, the force thus found is denoted by $\mathfrak{F}$, and is independent of free magnetism on the walls of the crevasse. If, however, the crevasse be perpendicular to the lines of force, there is a full development of free magnetism $(\Im)$ upon the walls, and the interior force is now $\mathfrak{B}$, equal to $\mathfrak{D}+4 \pi \mathfrak{Y}$. In the estimation of $\mathfrak{S}$ (as well as of $\mathfrak{B}$ ) the influence of all free magnetism, not dependent upon the imaginary interruption of continuity, is of course to be included. On this account the value of $\mathfrak{5}$ in the interior, and even at the centre, of a bar of iron placed in an otherwise uniform magnetic field, is greatly reduced, unless the length of the bar be a very large multiple of the diameter.

Experiment shows that the relation of $\mathfrak{B}$ to $\mathfrak{S}$ is not of a determinate character. In a cycle of operations, during which $\mathfrak{S}$ is first increased, and is afterwards brought back to its original value, the induction $\mathfrak{B}$ is always greater on the descending than on the ascending course. This phenomenon, which is exemplified familiarly by the retention of magnetism in a bar after withdrawal of the magnetizing force, is

* Communicated by the Author.

$\dagger$ "Experimental Researches in Magnetism," vol. clxxvi. partii. p. 523.

$\ddagger$ "Magnetization of Iron," ibid. p. 455 . 
called by Ewing hysteresis. The accompanying curve ABCDEFGA (fig. 1) is copied from one given by him as applicable to very soft iron, conducted round a cycle from strong negative to strong positive magnetization and back again. The "residual magnetism" or " retentiveness" $(\mathrm{OE})$ amounts to a large fraction (sometimes to 93 per cent.) of the maximum.

The work spent in carrying the iron round a magnetic cycle is represented by $-\int \Im d \mathfrak{S}$, as was first shown by Warburg *, who supposes the magnetic force operative upon the soft iron to be due to permanent magnets, and variable with their position. The work required to carry the permanent magnets through the proposed cycle of motions is then

Fig. 1.

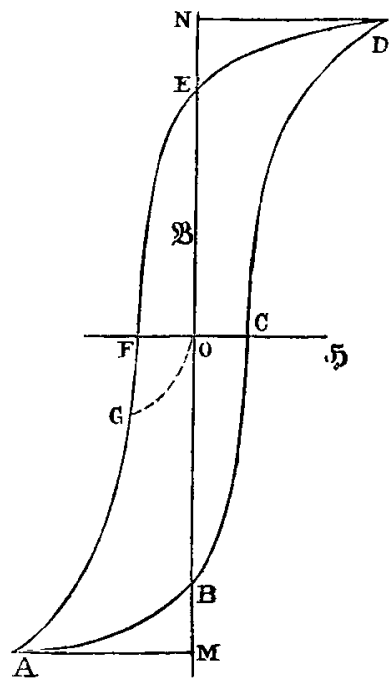
proved to have the above written value, applicable to the unit of volume of the soft iron. If $\Im$ were proportional to $\mathfrak{F}$, or even related to it in any determinate manner, the integral would vanish; but on account of hysteresis it has a finite value.

So long as we limit our attention to complete cycles, we may write indifferently $-\int \mathfrak{Y} d \mathfrak{S}$, or $-\frac{1}{4 \pi} \int \mathfrak{B} d \mathfrak{S}$, since $\int \mathfrak{S} d \mathfrak{E}$ vanishes. Again, under the same restriction,

$$
-\int \Im d \mathfrak{S}=+\int \mathfrak{S} d \Im=\frac{1}{4 \pi} \int \mathfrak{S} d \mathfrak{B} .
$$

When, however, we wish to consider incomplete cycles, especially with reference to the behaviour of soft iron, it is more suitable to take $\Im$ as independent variable. We are led naturally to this form if we suppose that, as in the more important practical applications, the varying magnetizing force is due to an electric current, upon which the magnetized iron reacts inductively $\dagger$.

In order to avoid the question of free polarity, we may

* Wied. Ann. xiii. p. 141 (1881).

$\dagger$ Hopkinson, l. c. p. 466. 
consider, first, a ring electro-magnet with an iron core, of length $l$ and section $\sigma$. If $n$ be the number of windings, the whole inductive electromotive force is $n \sigma . d \mathfrak{B}$, and the element of work is $n \sigma \mathrm{C} . d \mathfrak{B}, \mathrm{C}$ being the current at the moment in question. But

$$
l \mathfrak{g}=4 \pi n \mathrm{C} ;
$$

so that the element of work is, per unit of volume of iron,

$$
\frac{1}{4 \pi} \mathfrak{S} d \mathfrak{B} \text {. }
$$

If we express $\mathfrak{B}$ in terms of $\mathfrak{S}$ and $\mathfrak{F}$, we have

$$
\frac{1}{4 \pi} \mathfrak{W} d \mathfrak{B}=\frac{1}{4 \pi} \mathfrak{S} d \mathfrak{S}+\mathfrak{S} d \mathfrak{\Im},
$$

of which the latter part is specially due to the iron. In practice the former part is small, and the distinction between

$$
\frac{1}{4 \pi} \mathfrak{S} d \mathfrak{B} \text { and } \mathfrak{S} d \mathfrak{F}
$$

may often be disregarded.

But it is by no means a matier of indifference whether we take $\mathfrak{B} d \mathfrak{S}$ or $\mathfrak{S} d \mathfrak{B}$. The difference between the two modes of reckoning may be exemplified in the case of iron already nearly "saturated," and exposed to an increasing force. Here $\int \mathfrak{B} d \mathfrak{S}$ is large, while $\int \mathfrak{S} d \mathfrak{B}$ is small ; so that the latter corresponds better with the changes which we suppose to be taking place in the iron, as well as to the circumstances of ordinary practice.

Let us now consider a little more closely the cycle of fig. 1 . From $\mathrm{A}$ to $\mathrm{B}, \mathfrak{F}$ is negative, while $d \mathfrak{B}$ is positive; so that along $\mathrm{AB}$ the inductive electromotive force is in aid of the current, and work is received from the iron of amount represented by the area $A B M$. From $B$ to $D, \mathfrak{g}$ is positive as well as $d \mathfrak{B}$, and work represented by BDNB may be supposed to be put into the iron. From $\mathrm{D}$ to $\mathrm{E}$, work, represented by $\mathrm{NED}$, is received from the iron, and from $\mathrm{E}$ to $\mathrm{A}$ work, represented by $\triangle M E$, is expended. From this we see that not only is work, represented by the area $A B C D E A$, dissipated in the complete cycle, but that at no part of the cycle is there more than an insignificant fraction of work recovered. The case is not one of a storing of energy recoverable with a small relative loss, but rather one of almost continuous dissipation.

And here the question is forced upon us, whether it is true, as is usually supposed, that the strong residual magnetism at

Phil. Mag. S. 5. Vol. 22. No. 135. August 1886. N 
$\mathrm{E}$ is really a store of energy. From the fact that the magnetism may be got rid of by very moderate tapping, we may infer, I admit, that some energy is necessarily dissipated in passing from the magnetized to the unmagnetized condition, but the dissipation may be exceedingly small ; and the argument is not conclusive, since the mechanical energy of the vibrations may be involved in the process. If we attempt to demagnetize the iron in a straightforward manner by the application of a reversed force, following the course indicated by EFGO, then, so far from recovering, we actually expend energy - that, namely, represented by the area EFGOE. For practical purposes, at any rate, it would seem that magnetized iron cannot be regarded as the seat of available energy.

The opposite opinion, which is widely entertained, appears to depend upon insufficient observance of the distinction, vital to this subject, between closed and unclosed magnetic circuits. It is not disputed that available energy accompanies the magnetization of a short bar of iron, but this is in virtue of the free polarity at the ends. The work stored is in fact that which might be obtained, were the bar flexible, by allowing the ends to approach one another, under their mutual attraction. When this operation is finished, so that the bar has become a ring, there is no longer any work to be got out of it, though it remains magnetized.

In further illustration of this matter, reference may be made to some interesting observations by Elphinstone and Vincent * on closed magnetic circuits. As is well known, the armature of a horseshoe electromagnet remains strongly attracted after cessation of the battery-current. If, even after a considerable interval of time, the coils of this electromagnet were connected with those of a second electromagnet also provided with an armature, and the first armature were then violently pulled away, attraction set in and persisted between the second armature and its electromagnet, the magnetism of the original circuit being as it were transferred to the second. Or, if a galvanometer were substituted for the second electromagnet, a deflection followed the forcible withdrawal of the armature. In these experiments the necessary energy is obtained, not from the magnetism of the closed circuit, but from the work done in opening it, that is in pulling away the armature.

These considerations lead me to differ from Prof. Ewing when he says $\uparrow:-$ " In connection with ' secondary generators' and induction-coils generally, the bearing of the first part of

* Proc. Roy. Soc. vol. xxx. p. 287 (1880).

+ l.c. $\$ 34$, p. 554 . 
this paper should be noted, as showing the enormous advantage which a ring-shaped core, or core forming a complete magnetic circuit, possesses over a short bar-core with ends. In an ordinary induction-coil, so long as the current in the primary circuit is merely made and broken, a short core is necessary, since a ring-core would lose but a small percentage of its magnetism at each brake, but where reversal of the magnetizing takes place, a core approximating to the condition of endlessness has an advantage in respect of power which fig. 3 makes obvious." I confess that I do not follow this. It seems to me, on the contrary, that a closed magnetic circuit is above all things to be avoided, as leading to waste of the greater part of the power transferred.

A like objection applies to the use of a closed electromagnet as a "throttle" in an alternate-current circuit.

When we know, as from Prof. Ewing's results, the behaviour of a given sample of iron under the influence of various forces $\$$ actually operative, we can deduce by means of Poisson's theory the magnetism assumed by ellipsoids of any shape in response to any uniform external force $\mathfrak{S}^{\prime}$. If $\Im$ bo the magnetization parallel to the axis of symmetry $(2 c)$, the demagnetizing effect of $\mathfrak{Y}$ is $\mathrm{N} \mathfrak{Y}$, where $\mathrm{N}$ is a numerical constant, a function of the eccentricity $(e)^{*}$. When the ellipsoid is of the ovary or elongated form,

$$
\begin{gathered}
a=b=\downarrow^{\prime}\left(1-e^{2}\right) c \\
\mathrm{~N}=4 \pi\left(\frac{1}{e^{2}}-1\right)\left(\frac{1}{2 e} \log \frac{1+e}{1-e}-1\right)
\end{gathered}
$$

becoming in the limiting case of the sphere $(e=0)$

$$
\mathrm{N}=\frac{4 \pi}{3} ;
$$

and at the other extreme of elongation assuming the form

$$
\mathrm{N}=4 \pi \frac{a^{2}}{c^{2}}\left(\log \frac{2 c}{a}-1\right)
$$

If the ellipsoid is of the planetary form,

and

$$
a=b=\frac{e}{\sqrt{ }\left(1-e^{2}\right)} \dagger
$$

$$
\mathrm{N}=4 \pi\left(\frac{1}{e^{2}}-\frac{\sqrt{ }\left(1-e^{2}\right.}{e^{3}} \sin ^{-1} e\right)
$$

* Maxwell's 'Electricity and Magnetism,' $\$ 438$.

+ There is here a slight variation from Maxwell's notation.

N 2 
In the case of a very flattened planetoid $(e=1), \mathrm{N}$ becomes in the limit equal to $4 \pi$.

The force actually operative upon the iron is formed by subtracting $\mathrm{N}\}$ from that externally imposed, so that

$$
\mathfrak{S}=\mathfrak{S}^{\prime}-\mathrm{N} \mathfrak{S} \text {; }
$$

and if from experiments on very elongated ellipsoids $(\mathrm{N}=0)$ we know the relation between $\mathfrak{S}$ and $\mathfrak{\Im}$, then the above equation gives us the relation between $\mathfrak{S}^{\prime}$ and $\Im$ for any proposed ellipsoid of finite elongation. If we suppose that $\mathfrak{S}$ is plotted as a function of $\mathfrak{\Im}$, we have only to add in the ordinates $\mathrm{N} . \checkmark$, proper to a straight line, in order to obtain the appropriate curve for $\mathfrak{S}^{\prime}$.

As an example, let as apply this method to deduce the behaviour of the soft iron of Ewing's fig. 2, when made into an ellipsoid whose polar axis is fifty times the equatorial axis, and carried round a cycle through strong positive and strong negative magnetism. We have

$$
\mathrm{N}=\frac{4 \pi}{50^{2}}\left\{\log _{e} 100-1\right\}=4 \pi \times \cdot 001442
$$

The curve ABC (fig. 2), traced from Prof. Ewing's, gives Fig. 2.

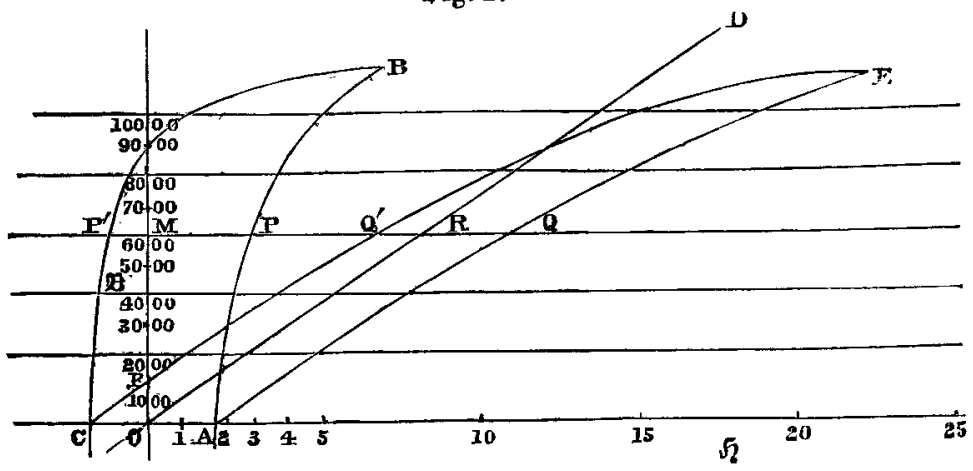

the relation between $\mathfrak{S}$ and $\mathfrak{P}$, the latter of which we may identify with $4 \pi \Im^{*}$. The equation of the straight line is

$$
\mathfrak{D}=\mathrm{N} \mathfrak{\Im}=\cdot 001442 \times 4 \pi \Im ;
$$

and with allowance for the different scales adopted for ordinates and abscissæ, is represented on the diagram by OD.

* The curve is symmetrical with respect to $O$ as centre, and $\mathfrak{S}$ is measured in C.G.S. units. 
In order to find the points $Q, Q^{\prime}$ appropriate to the ellipsoid $(50: 1)$ from $P, \mathrm{P}^{\prime}$, we have merely to measure $P Q, \mathrm{P}^{\prime} \mathrm{Q}^{\prime}$ equal to RM. We thus obtain the curve $A Q E Q^{\prime} F C$, on which the points of zero magnetization are the same as on the original curve ${ }^{*}$. We see that a much stronger field is now required to produce the higher degrees of magnetization, and that there is less hysteresis-the magnetic state is more nearly a definite function of the external field. A similar construction might be used reversely to pass from observed results relative to ellipsoids of moderate elongation to the curve appropriate to ellipsoids of infinite elongation, on which alone we can base our views of the real character of magnetic media.

Prof. Ewing has traced by experiment the influence of various degrees of elongation on the magnetism of cylindrical rods. Results of this kind are exhibited in his fig. 3, but they are not strictly comparable with those obtained above, not only because the latter relate to ellipsoids, but also on account of the different character of the magnetic operations represented. His curves begin at a condition of zero field and zero magnetization.

The work expended in producing a small change of magnetization of the ellipsoid, acted upon by a uniform field, is $\mathfrak{S}^{\prime} d \Im$ simply per unit of volume. This we may see, perhaps most easily, by supposing the iron to be replaced by an electric current of equal magnetic moment. The element of work done then depends upon the coefficient of mutual induction $M$ of the two circuits, and $M$ may be regarded as the number of lines of force due to the original current which pass through the fictitious circuit. The whole work is thins

$$
\begin{aligned}
\int \mathfrak{D}^{\prime} d \mathfrak{I} & =\int \mathfrak{S} d \mathfrak{\Im}+\mathrm{N} \int \mathfrak{I} d \mathfrak{\Im} \\
& =\int \mathfrak{S} d \mathfrak{\Im}+\frac{1}{2} \mathrm{~N} \mathfrak{\Im}^{2},
\end{aligned}
$$

if we reckon from the condition of zero magnetization. The first part is that already considered, and shown to bo almost entirely wasted; the second, which in most cases of open magnetic circuits is much the larger, is completely recovered when the iron is demagnetized.

Thus in fig. 2, since $\mathrm{QQ}^{\prime}=\mathrm{PP}^{\prime}$, the areas of the two curves are the same, which indicates that the same amount of work is dissipated in a complete cycle. But the work absorbed during one part and restored during the remainder of the

* Dr. Hopkinsen (loc. cit. p. 465) has already applied this method to the determination of the particular point $F$, indicative of the residual magnetism in the ellipsoid, when the external force is withdrawn. 
cycle is much greater in the case of $\mathrm{AEC}$, corresponding to the ellipsoid of moderate elongation.

The coefficient $\mathrm{N}$ reaches its maximum when the ellipsoid is very oblate. In this case

$$
\int \mathfrak{S}^{\prime} d \mathfrak{\Im}=\int \mathfrak{S} d \Im+2 \pi \mathfrak{\Im}^{2}
$$

which is applicable to large plates magnetized perpendicularly to their surfaces. This is the form to which the iron must be reduced in order that a given magnetization of a given volume may store* the largest amount of energy. In this case the energy is nearly all recoverable; but we must remember that no practicable field would carry the magnetization very far.

In the theory of alternating currents the neighbourhood of of iron is often treated as if its only effect were to increase the self and mutual induction of the circuits. A writer conversant with experiment usually guards himself by a reference to the currents induced in the iron considered as a conductor. The latter effect may be in great measure eliminated by a proper subdivision of the iron, with intervening non-conducting strata; but a glance at fig. 2 shows at once that, apart altogether from internal currents, the influence of the iron is of a more complicated character. If the curve connecting $\mathfrak{Y}$ (or $\mathfrak{B}$ ) and $\mathfrak{S}$ were a straight line, the same on the upward as on the downward course, then the presence of iron would simply increase the self-induction. When the iron constitutes a closed magnetic circuit, this is very far from being true. Indeed it would be nearer the mark to say that the iron increases the apparent resistance of the electric circuit, leaving the self-induction unchanged. In so far as the curve of fig. 2 can be identified with an ellipse, the reaction of the iron can be represented as equivalent to a change in the apparent resistance and self-induction of the circuit. Which of the two is the more important depends somewhat upon the other circumstances of the case ; but with closed electromagnets the magnetic work dissipated during the period (corresponding to increased resistance) is always greater than the work spent during one part and recovered during the remainder of the period (corresponding to increased self-induction). On this account, the resistance of an iron wire to variable currents is greater than to steady currents, even though the current be constrained to be uniformly distributed over the section. In the absence of such constraint, the resistance undergoes a further increase in consequence of the tendency of the cur-

* It is not meant here to imply that the energy is resident in the iron. 
rent to concentrate itself towards the exterior*. In general both causes must cooperate to produce an apparent increase of resistance to variable currents.

When the magnetic circuits are open, as with bars of iron of moderate length, the reaction of the iron manifests itself mainly as increased self-induction. This happens also in the case of closed magnetic circuits, when the magnetic changes are very small.

In general, since the curve of fig. 2 differs widely from an ellipse, the reaction of the iron cannot be fully represented as equivalent to a change in the resistance and self-induction of the magnetizing circuit. In any case of strict periodicity the reaction may, however, be analyzed, in accordance with Fourier's theorem, into harmonic components with periods which are submultiples of the original period. The neighbourhood of iron may thus introduce overtones into what would otherwise be a simple sound.

Terling Place, Witham, Essex, July 4.

XXII. On the Salts of Tetrethylphosphonium and their Decomposition by Heat. By Prof. E. A. LetTs, Ph.D., \& c., Queen's College, Belfast, and Norman Collie, Ph.D., Science Lecturer, The Ladies' College, Cheltenham $\dagger$.

$0 \mathrm{NE}$ of us (in conjunction with another chemist) has already pointed out the very striking analogies which exist between the elements phosphorus and sulphur and their compounds $\ddagger$, and we were anxious to continue our experiments in connection with this question.

The action of heat on methyl-sulphine compounds has been studied by Crum Brown and Blaikie §, who have shown that they decompose in a perfectly simple and definite manner. $\mathrm{We}$ were accordingly desirous of ascertaining whether the analogous phosphorus derivatives, namely the salts of tetrethylphosphonium, would behave similarly.

We had already investigated the action of heat on some of the salts of tetrabenzylphosphonium $\|$, but the results were

* "On the Self-induction and Resistance of Straight Conductors," Phil. Mag. vol. xxi. (1886) p. 388.

$\dagger$ Communicated by the Authors.

† Crum Brown and Letts, Trans. Roy. Soc. Edin. vol. xxviii. p. 571; and Letts, Trans. Roy. Soc. Edin. vol. xxviii. p. 583.

\$ Crum Brown and Blaikie, Journal f. prakt. Chemie [2] xxiii. p. 395.

if Letts and Collie, Trans. Roy. Soc. Edin. vol. xxx. part i. p. 181. 